\title{
Existence of Mild Solutions for the Elastic Systems with Structural Damping in Banach Spaces
}

\author{
Hongxia Fan, ${ }^{1,2}$ Yongxiang $\mathrm{Li}^{2}$ and Pengyu Chen ${ }^{2}$ \\ ${ }^{1}$ Department of Mathematics, Lanzhou Jiaotong University, Lanzhou 730070, China \\ ${ }^{2}$ Department of Mathematics, Northwest Normal University, Lanzhou 730070, China
}

Correspondence should be addressed to Hongxia Fan; lzfanhongxia@163.com

Received 2 November 2012; Revised 6 January 2013; Accepted 16 January 2013

Academic Editor: Ferenc Hartung

Copyright (C) 2013 Hongxia Fan et al. This is an open access article distributed under the Creative Commons Attribution License, which permits unrestricted use, distribution, and reproduction in any medium, provided the original work is properly cited.

This paper deals with the existence and uniqueness of mild solutions for a second order evolution equation initial value problem in a Banach space, which can model an elastic system with structural damping. The discussion is based on the operator semigroups theory and fixed point theorem. In addition, an example is presented to illustrate our theoretical results.

\section{Introduction}

Our aim in this paper is to study the existence and uniqueness of mild solutions for the semilinear elastic system with structural damping

$$
\begin{gathered}
\ddot{u}(t)+\rho \mathscr{A} \dot{u}(t)+\mathscr{A}^{2} u(t)=f(t, u(t)), \quad 0<t<a, \\
u(0)=x_{0}, \quad \dot{u}(0)=y_{0}
\end{gathered}
$$

in a Banach space $\mathbb{X}$, where - means $d / d t, \rho \geq 2$ is a constant; $\mathscr{A}: D(\mathscr{A}) \subset \mathbb{X} \rightarrow \mathbb{X}$ is a closed linear operator and $-\mathscr{A}$ generates a $C_{0}$-semigroup $T(t)(t \geq 0)$ on $\mathbb{X} ; f \in C([0, a] \times$ $\mathbb{X}, \mathbb{X}), x_{0} \in D(\mathscr{A}), y_{0} \in \mathbb{X}$.

In 1982, Chen and Russell [1] investigated the following linear elastic system described by the second order equation

$$
\ddot{u}(t)+B \dot{u}(t)+A u(t)=0
$$

in a Hilbert space $H$ with inner $(\cdot, \cdot)$, where $A$ (the elastic operator) and $B$ (the damping operator) are positive definite selfadjoint operators in $H$. They reduced (2) to the first order equation in $H \times H$

$$
\frac{d}{d t}\left(\begin{array}{c}
A^{1 / 2} u \\
\dot{u}
\end{array}\right)=\left(\begin{array}{cc}
0 & A^{1 / 2} \\
-A^{1 / 2} & -B
\end{array}\right)\left(\begin{array}{c}
A^{1 / 2} u \\
\dot{u}
\end{array}\right)
$$

Let $V=\mathscr{D}\left(A^{1 / 2}\right), \quad \mathscr{H}=V \times H$ with the naturally induced inner products. Then, (2) is equivalent to the first order equation in $\mathscr{H}$

$$
\frac{d}{d t}\left(\begin{array}{l}
u \\
\dot{u}
\end{array}\right)=\mathscr{A}_{B}\left(\begin{array}{l}
u \\
\dot{u}
\end{array}\right)
$$

where

$$
\begin{gathered}
\mathscr{A}_{B}=\left(\begin{array}{cc}
0 & I \\
-A & -B
\end{array}\right), \\
D\left(\mathscr{A}_{B}\right)=D(A) \times\left[D\left(A^{1 / 2}\right) \cap D(B)\right] .
\end{gathered}
$$

Chen and Russell [1] conjectured that $\mathscr{A}_{B}$ is the infinitesimal generator of an analytic semigroup on $\mathscr{H}$ if

$$
D\left(A^{1 / 2}\right) \subset D(B)
$$

and either of the following two inequalities holds for some $\beta_{1}, \beta_{2}>0$ :

$$
\begin{gathered}
\beta_{1}\left(A^{1 / 2} v, v\right) \leq(B v, v) \leq \beta_{2}\left(A^{1 / 2} v, v\right), \quad v \in D\left(A^{1 / 2}\right) \\
\beta_{1}(A v, v) \leq\left(B^{2} v, v\right) \leq \beta_{2}(A v, v), \quad v \in D(A) .
\end{gathered}
$$

In the same paper they obtained some results in this direction. The complete proofs of the two conjectures were given by 
Huang $[2,3]$. Then, other sufficient conditions for $\mathscr{A}_{B}$ or its closure $\overline{\mathscr{A}_{B}}$ to generate an analytic or differentiable semigroup on $\mathscr{H}$ were discussed in [4-10], by choosing $B$ to be an operator comparable with $A^{\alpha}$ for $0<\alpha \leq 1$, based on an explicit matrix representation of the resolvent operator of $\mathscr{A}_{B}$ or $\overline{\mathscr{A}_{B}}$.

However, so far as we know, among the previous works, little is concerned with an elastic system with structural damping in a Banach space. Motivated by previous works, in this paper, we investigate the existence and uniqueness of mild solutions for the elastic system (1) in a frame of Banach spaces. To this end, we firstly introduce the concept of mild solutions for system (1), which is based on the discussion about associated linear system. Secondly, we prove the existence and uniqueness of mild solutions for the semilinear elastic system (1) in a Banach space $\mathbb{X}$.

The paper is organized as follows. In Section 2, we discuss the associated linear elastic system and give its definition of mild solutions. In Section 3, we study the existence and uniqueness of mild solutions for the semilinear elastic system (1). An example to illustrate our theoretical results is given in Section 4.

\section{Preliminaries on Linear Elastic Systems}

Let $\mathbb{X}$ be a Banach space, we consider the linear elastic system with structural damping

$$
\begin{gathered}
\ddot{u}(t)+\rho \mathscr{A} \dot{u}(t)+\mathscr{A}^{2} u(t)=h(t), \quad 0<t<a, \\
u(0)=x_{0}, \quad \dot{u}(0)=y_{0},
\end{gathered}
$$

where - means $d / d t, \rho \geq 2$ is a constant; $\mathscr{A}: D(\mathscr{A}) \subset \mathbb{X} \rightarrow \mathbb{X}$ is a closed linear operator, and $-\mathscr{A}$ generates a $C_{0}$-semigroup $T(t)(t \geq 0)$ on $\mathbb{X} ; h:[0, a] \rightarrow \mathbb{X}, x_{0} \in D(\mathscr{A}), y_{0} \in \mathbb{X}$.

For the second order evolution equation

$$
\ddot{u}(t)+\rho \mathscr{A} \dot{u}(t)+\mathscr{A}^{2} u(t)=h(t),
$$

it has the following decomposition

$$
\left(\frac{\partial}{\partial t}+\sigma_{1} \mathscr{A}\right)\left(\frac{\partial}{\partial t}+\sigma_{2} \mathscr{A}\right) u=h(t) .
$$

That is,

$$
\frac{\partial^{2} u}{\partial t^{2}}+\left(\sigma_{1}+\sigma_{2}\right) \mathscr{A} \frac{\partial u}{\partial t}+\sigma_{1} \sigma_{2} \mathscr{A}^{2} u=h(t)
$$

It follows from (9) and (11) that

$$
\sigma_{1}+\sigma_{2}=\rho, \quad \sigma_{1} \sigma_{2}=1 .
$$

By (12), we have
(i) if $\rho>2, \quad$ then $\sigma_{1}=\frac{\rho+\sqrt{\rho^{2}-4}}{2}, \quad \sigma_{2}=\frac{\rho-\sqrt{\rho^{2}-4}}{2}$,
(ii) if $\rho=2, \quad$ then $\sigma_{1}=\sigma_{2}=1$.

Let

$$
\frac{\partial u}{\partial t}+\sigma_{2} \mathscr{A} u=v(t), \quad 0 \leq t \leq a
$$

which means

$$
v_{0}:=v(0)=y_{0}+\sigma_{2} \mathscr{A} x_{0}
$$

So we reduce the linear elastic system (8) to the following two abstract Cauchy problems in Banach space $\mathbb{X}$ :

$$
\begin{gathered}
\frac{\partial v}{\partial t}+\sigma_{1} \mathscr{A} v=h(t), \quad 0<t<a, \\
v(0)=v_{0}, \\
\frac{\partial u}{\partial t}+\sigma_{2} \mathscr{A} u=v(t), \quad 0<t<a, \\
u(0)=x_{0} .
\end{gathered}
$$

It is clear that (16) and (17) are linear inhomogeneous initial value problems for $-\sigma_{1} \mathscr{A}$ and $-\sigma_{2} \mathscr{A}$, respectively. Since $-\mathscr{A}$ is the infinitesimal generator of $C_{0}$-semigroup $T(t)(t \geq 0)$. Furthermore, for any $\rho \geq 2$, (13) yield $\sigma_{1}>0, \sigma_{2}>0$. Thus, by operator semigroups theory [11], $-\sigma_{1} \mathscr{A}$ and $-\sigma_{2} \mathscr{A}$ are infinitesimal generators of $C_{0}$-semigroups, which implies initial value problems (16) and (17) are well-posed.

Throughout this paper, we assume that $-\sigma_{1} \mathscr{A}$ and $-\sigma_{2} \mathscr{A}$ generate $C_{0}$-semigroups $S_{1}(t)(t \geq 0)$ and $S_{2}(t)(t \geq 0)$ on $\mathbb{X}$, respectively. Note that $\sigma_{1}>0, \sigma_{2}>0$ and $-\mathscr{A}$ is the infinitesimal generator of $C_{0}$-semigroup $T(t)(t \geq 0)$. It follows that

$$
S_{1}(t)=T\left(\sigma_{1} t\right), \quad S_{2}(t)=T\left(\sigma_{2} t\right), \quad t \geq 0 .
$$

It is well known $[12$, Chapter 4$]$, when $h \in L^{1}([0, a], \mathbb{X})$, the linear initial value problem (16) has a mild solution $v$ given by

$$
v(t)=S_{1}(t) v_{0}+\int_{0}^{t} S_{1}(t-s) h(s) d s
$$

Similarly, if $v \in C([0, a], \mathbb{X})$, then the mild solution of the linear initial value problem (17) expressed by

$$
u(t)=S_{2}(t) x_{0}+\int_{0}^{t} S_{2}(t-s) v(s) d s
$$

Substituting (19) into (20), we get

$$
\begin{aligned}
u(t)= & S_{2}(t) x_{0}+\int_{0}^{t} S_{2}(t-s) S_{1}(s) v_{0} d s \\
& +\int_{0}^{t} \int_{0}^{s} S_{2}(t-s) S_{1}(s-\tau) h(\tau) d \tau d s .
\end{aligned}
$$

From the argument above, we obtain the following corollary.

Corollary 1. If $\in \in L^{1}([0, a], \mathbb{X})$, then the initial value problem (8) has at most one solution. If it has a solution, this solution is given by (21). 
For every $h \in L^{1}([0, a], \mathbb{X})$, the right-hand side of $(21)$ is a continuous function on $[0, a]$. It is natural to consider it as a generalized solution of (8) even it is not differentiable and dose not strictly satisfy the equation. We therefore define the following.

Definition 2. Let $-\mathscr{A}$ is the infinitesimal generator of $C_{0}$ semigroup $T(t)(t \geq 0)$. Then a continuous solution $u(t)$ of the integral equation

$$
\begin{aligned}
u(t)= & S_{2}(t) x_{0}+\int_{0}^{t} S_{2}(t-s) S_{1}(s) v_{0} d s \\
& +\int_{0}^{t} \int_{0}^{s} S_{2}(t-s) S_{1}(s-\tau) h(\tau) d \tau d s
\end{aligned}
$$

is said to be a mild solution of the initial value problem (8). Where $S_{1}(t)(t \geq 0), S_{2}(t)(t \geq 0)$ were defined in (18) and $v_{0}$ was specified in (15).

\section{Main Results}

Let $C(J, \mathbb{X})$ be the Banach space of all continuous functions $u: J \rightarrow \mathbb{X}$ with norm $\|u\|_{C}=\max _{s \in J}\|u(s)\|, J=[0, a]$. Let $\mathscr{L}(\mathbb{X})$ be the Banach space of all linear and bounded operators on $\mathbb{X}$. Note that $S_{1}(t)(t \geq 0)$ and $S_{2}(t)(t \geq 0)$ are $C_{0}$-semigroups on $\mathbb{X}$. Thus, there exist $M_{1} \geq 1$ and $M_{2} \geq 1$ such that

$$
M_{1}=\sup _{t \in J}\left\|S_{1}(t)\right\|_{\mathscr{L}(\mathbb{X})}, \quad M_{2}=\sup _{t \in J}\left\|S_{2}(t)\right\|_{\mathscr{L}(\mathbb{X})} .
$$

In what follows, we firstly give the definition of a mild solution for the initial value problem (1) below.

Definition 3. Let $-\mathscr{A}$ is the infinitesimal generator of $C_{0^{-}}$ semigroup $T(t)(t \geq 0)$. Then a continuous solution $u(t)$ of the integral equation

$$
\begin{aligned}
u(t)= & S_{2}(t) x_{0}+\int_{0}^{t} S_{2}(t-s) S_{1}(s) v_{0} d s \\
& +\int_{0}^{t} \int_{0}^{s} S_{2}(t-s) S_{1}(s-\tau) f(\tau, u(\tau)) d \tau d s
\end{aligned}
$$

is said to be a mild solution of the initial value problem (1). Where $S_{1}(t)(t \geq 0), S_{2}(t)(t \geq 0)$ were defined in (18) and $v_{0}$ was specified in (15).

Secondly, we consider the existence and uniqueness of mild solutions for (1). To this end, we make the following assumptions:

(H1) $f:[0, a] \times \mathbb{X} \rightarrow \mathbb{X}$ be continuous and there exists $L>0$, such that

$$
\begin{aligned}
& \left\|f\left(t, u_{2}\right)-f\left(t, u_{1}\right)\right\| \\
& \quad \leq L\left\|u_{2}-u_{1}\right\|, \quad t \in[0, a], u_{1}, u_{2} \in \mathbb{X} .
\end{aligned}
$$

(H2) $f:[0, a] \times \mathbb{X} \rightarrow \mathbb{X}$ be continuous and there exists a positive function $\mu \in L^{\infty}\left(J, \mathbb{R}^{+}\right)\left(\mathbb{R}^{+}=[0,+\infty)\right)$ such that

$$
\|f(t, u)\| \leq \mu(t), \quad t \in[0, a], u \in \mathbb{X} .
$$

(H3) The $C_{0}$-semigroup $T(t)(t \geq 0)$ is compact for $t>0$.

Theorem 4. Assume that $(H 1)$ holds, $-\mathscr{A}$ is the infinitesimal generator of $C_{0}$-semigroup $T(t)(t \geq 0)$. Then for every $x_{0} \in$ $D(\mathscr{A}), y_{0} \in \mathbb{X}$ and $\rho \geq 2$, the initial value problem (1) has a unique mild solution $u \in C([0, a], \mathbb{X})$.

Proof. Define the operator $Q: C(J, \mathbb{X}) \rightarrow C(J, \mathbb{X})$ by

$$
\begin{aligned}
(Q u)(t)= & S_{2}(t) x_{0}+\int_{0}^{t} S_{2}(t-s) S_{1}(s) v_{0} d s \\
& +\int_{0}^{t} \int_{0}^{s} S_{2}(t-s) S_{1}(s-\tau) f(\tau, u(\tau)) d \tau d s .
\end{aligned}
$$

It is obvious that the mild solution of the initial value problem (1) is equivalent to the fixed point of $Q$.

For any $u_{1}, u_{2} \in C(J, \mathbb{X}),(23),(27)$, and (H1) yield

$$
\begin{aligned}
& \left\|\left(Q u_{2}\right)(t)-\left(Q u_{1}\right)(t)\right\| \\
& \leq \int_{0}^{t} \int_{0}^{s}\left\|S_{2}(t-s)\right\|_{\mathscr{L}(\mathbb{X})}\left\|S_{1}(s-\tau)\right\|_{\mathscr{L}(\mathbb{X})} \\
& \quad \times\left\|f\left(\tau, u_{2}(\tau)\right)-f\left(\tau, u_{1}(\tau)\right)\right\| d \tau d s \\
& \leq L M_{1} M_{2} \int_{0}^{t} \int_{0}^{s}\left\|u_{2}(\tau)-u_{1}(\tau)\right\| d \tau d s \\
& \leq L M_{1} M_{2} \int_{0}^{t} \int_{0}^{s}\left\|u_{2}-u_{1}\right\|_{C} d \tau d s \\
& \leq \frac{L M_{1} M_{2} a^{2}}{2}\left\|u_{2}-u_{1}\right\|_{C} .
\end{aligned}
$$

Using (27), (28), and induction on $n$ it follows easily that

$$
\left\|Q^{n} u_{2}(t)-Q^{n} u_{1}(t)\right\| \leq \frac{\left(L M_{1} M_{2} a^{2}\right)^{n}}{(2 n) !}\left\|u_{2}-u_{1}\right\|_{C} .
$$

Hence

$$
\left\|Q^{n} u_{2}-Q^{n} u_{1}\right\|_{C} \leq \frac{\left(L M_{1} M_{2} a^{2}\right)^{n}}{(2 n) !}\left\|u_{2}-u_{1}\right\|_{C} .
$$

Since

$$
\frac{\left(L M_{1} M_{2} a^{2}\right)^{n}}{(2 n) !} \longrightarrow 0 \text { as } n \longrightarrow \infty .
$$

Thus, for $n$ large enough $\left(L M_{1} M_{2} a^{2}\right)^{n} /(2 n) !<1$ and by well known extension of the contraction mapping principle, $Q$ has a unique fixed point $u \in C([0, a], \mathbb{X})$. This fixed point is the desired solution of the integral equation (24).

Theorem 5. Suppose that assumptions (H2) and (H3) hold. Then for every $x_{0} \in D(\mathscr{A}), y_{0} \in \mathbb{X}$ and $\rho \geq 2$, the initial value problem (1) has at least one mild solution $u \in C([0, a], \mathbb{X})$. 
Proof. Define the operator $Q: C(J, \mathbb{X}) \rightarrow C(J, \mathbb{X})$ as (27) and choose $r>0$ such that

$$
r \geq M_{2}\left\|x_{0}\right\|+M_{1} M_{2} a\left\|v_{0}\right\|+M_{1} M_{2} a^{2}\|\mu\|_{L^{\infty}\left(J, \mathbb{R}^{+}\right)} .
$$

Let $B_{r}=\left\{u \in C(J, \mathbb{X}):\|u\|_{C} \leq r\right\}$. We proceed in two main steps.

Step 1. We show that $Q\left(B_{r}\right) \subset B_{r}$. For that, let $u \in B_{r}$. Then for $t \in J$, we have

$$
\begin{aligned}
\|(Q u)(t)\| \leq & \left\|S_{2}(t) x_{0}\right\|+\left\|\int_{0}^{t} S_{2}(t-s) S_{1}(s) v_{0} d s\right\| \\
& +\left\|\int_{0}^{t} \int_{0}^{s} S_{2}(t-s) S_{1}(s-\tau) f(\tau, u(\tau)) d \tau d s\right\|,
\end{aligned}
$$

which according to (H2) and (23) gives

$$
\begin{aligned}
\|(Q u)(t)\| \leq & M_{2}\left\|x_{0}\right\|+M_{1} M_{2} a\left\|v_{0}\right\| \\
& +M_{1} M_{2} a^{2}\|\mu\|_{L^{\infty}\left(J, \mathbb{R}^{+}\right)} .
\end{aligned}
$$

In view of the choice of $r$, we obtain

$$
\|Q u\|_{C} \leq r .
$$

Step 2. We prove that $Q$ is completely continuous. Note that $f$ : $u \rightarrow f(\cdot, u(\cdot))$ is a continuous mapping from $B_{r}$ to $C(J, \mathbb{X})$. Thus, $Q: B_{r} \rightarrow B_{r}$ is continuous. Next, we show that $Q$ is compact. To this end, we use the Ascoli-Arzela's theorem. For that, we first prove that $\left\{(Q u)(t): u \in B_{r}\right\}$ is relatively compact in $\mathbb{X}$, for $t \in J$. Obviously, $\left\{(Q u)(0): u \in B_{r}\right\}$ is compact.

Let $t \in(0, a]$. For each $\epsilon \in(0, t)$ and $u \in B_{r}$, we define the operator $Q_{\epsilon}$ by

$$
\begin{aligned}
\left(Q_{\epsilon} u\right)( & \\
= & S_{2}(t) x_{0}+\int_{0}^{t-\epsilon} S_{2}(t-s) S_{1}(s) v_{0} d s \\
& +\int_{0}^{t-\epsilon} \int_{0}^{s} S_{2}(t-s) S_{1}(s-\tau) f(\tau, u(\tau)) d \tau d s \\
= & S_{2}(t) x_{0}+S_{2}(\epsilon) \int_{0}^{t-\epsilon} S_{2}(t-\epsilon-s) S_{1}(s) v_{0} d s \\
& +S_{2}(\epsilon) \int_{0}^{t-\epsilon} \int_{0}^{s} S_{2}(t-\epsilon-s) S_{1}(s-\tau) \\
& \times f(\tau, u(\tau)) d \tau d s .
\end{aligned}
$$

Then the sets $\left\{\left(Q_{\epsilon} u\right)(t): u \in B_{r}\right\}$ are relatively compact in $\mathbb{X}$ since by $(H 3)$ and (18), the semigroup $S_{2}(t)(t \geq 0)$ is compact for $t>0$ on $\mathbb{X}$. Moreover, using (23) and (H2) we have

$$
\begin{aligned}
\left\|(Q u)(t)-\left(Q_{\epsilon} u\right)(t)\right\| \\
\leq\left\|\int_{t-\varepsilon}^{t} S_{2}(t-s) S_{1}(s) v_{0} d s\right\| \\
\quad+\left\|\int_{t-\varepsilon}^{t} \int_{0}^{s} S_{2}(t-s) S_{1}(s-\tau) f(\tau, u(\tau)) d \tau d s\right\| \\
\leq M_{1} M_{2}\left\|v_{0}\right\| \epsilon+M_{1} M_{2} a\|\mu\|_{L^{\infty}\left(J, \mathbb{R}^{+}\right)} \epsilon .
\end{aligned}
$$

Therefore, the set $\left\{(Q u)(t): u \in B_{r}\right\}$ is relatively compact in $\mathbb{X}$ for all $t \in(0, a]$ and since it is compact at $t=0$ we have the relatively compactness in $\mathbb{X}$ for all $t \in J$.

Now, let us prove that $Q\left(B_{r}\right)$ is equicontinuous. For $0 \leq$ $t_{1}<t_{2} \leq a$, we have

$$
\begin{aligned}
& \left\|(Q u)\left(t_{2}\right)-(Q u)\left(t_{1}\right)\right\| \\
& \leq\left\|S_{2}\left(t_{2}\right) x_{0}-S_{2}\left(t_{1}\right) x_{0}\right\| \\
& \quad+\left\|\int_{0}^{t_{1}}\left[S_{2}\left(t_{2}-s\right)-S_{2}\left(t_{1}-s\right)\right] S_{1}(s) v_{0} d s\right\| \\
& +\left\|\int_{t_{1}}^{t_{2}} S_{2}\left(t_{2}-s\right) S_{1}(s) v_{0} d s\right\| \\
& +\| \int_{0}^{t_{1}} \int_{0}^{s}\left[S_{2}\left(t_{2}-s\right)-S_{2}\left(t_{1}-s\right)\right] \\
& \quad+\left\|\int_{t_{1}}^{t_{2}} \int_{0}^{s} S_{2}\left(t_{2}-s\right) S_{1}(s-\tau) f(\tau, u(\tau)) d \tau d s\right\| \\
& :=I_{1}+I_{2}+I_{3}+I_{4}+I_{5},
\end{aligned}
$$

where

$$
\begin{aligned}
& I_{1}=\left\|S_{2}\left(t_{2}\right) x_{0}-S_{1}\left(t_{1}\right) x_{0}\right\|, \\
& I_{2}=\left\|\int_{0}^{t_{1}}\left[S_{2}\left(t_{2}-s\right)-S_{2}\left(t_{1}-s\right)\right] S_{1}(s) v_{0} d s\right\|, \\
& I_{3}=\left\|\int_{t_{1}}^{t_{2}} S_{2}\left(t_{2}-s\right) S_{1}(s) v_{0} d s\right\|, \\
& I_{4}=\| \int_{0}^{t_{1}} \int_{0}^{s}\left[S_{2}\left(t_{2}-s\right)-S_{2}\left(t_{1}-s\right)\right] \\
& \quad \times S_{1}(s-\tau) f(\tau, u(\tau)) d \tau d s \|, \\
& I_{5}=\left\|\int_{t_{1}}^{t_{2}} \int_{0}^{s} S_{2}\left(t_{2}-s\right) S_{1}(s-\tau) f(\tau, u(\tau)) d \tau d s\right\| .
\end{aligned}
$$


In fact, $I_{1}, I_{2}, I_{3}, I_{4}$ and $I_{5}$ tend to 0 independently of $u \in B_{r}$ when $t_{2}-t_{1} \rightarrow 0$.

Note that the function $S_{2}(t) x_{0}$ is continuous for $t \geq 0$. Thus, $S_{2}(t) x_{0}$ is uniformly continuous on $[0, a]$ and thus $\lim _{t_{2}-t_{1} \rightarrow 0} I_{1}=0$.

From (23) and (H2), we have

$$
\begin{aligned}
I_{2} \leq & \int_{0}^{t_{1}}\left\|S_{2}\left(t_{2}-s\right)-S_{2}\left(t_{1}-s\right)\right\|_{\mathscr{L}(\mathbb{X})} \\
& \times\left\|S_{1}(s)\right\|_{\mathscr{L}(\mathbb{X})}\left\|v_{0}\right\| d s \\
\leq & M_{1}\left\|v_{0}\right\| \int_{0}^{a}\left\|S_{2}\left(t_{2}-t_{1}+\tau\right)-S_{2}(\tau)\right\|_{\mathscr{L}(\mathbb{X})} d \tau . \\
I_{4} \leq & \int_{0}^{t_{1}} \int_{0}^{s}\left\|S_{2}\left(t_{2}-s\right)-S_{2}\left(t_{1}-s\right)\right\|_{\mathscr{L}(\mathbb{X})} \\
& \times\left\|S_{1}(s-\tau)\right\|_{\mathscr{L}(\mathbb{X})}\|f(\tau, u(\tau))\| d \tau d s \\
\leq & M_{1} a\|\mu\|_{L^{\infty}\left(J, \mathbb{R}^{+}\right)} \\
& \times \int_{0}^{a}\left\|S_{2}\left(t_{2}-t_{1}+\tau\right)-S_{2}(\tau)\right\|_{\mathscr{L}(\mathbb{X})} d \tau .
\end{aligned}
$$

Let $\phi(\tau)=S_{2}\left(t_{2}-t_{1}+\tau\right)-S_{2}(\tau)$. By the compactness of $T(\cdot)$ and (18), we can easily conclude that $S_{2}(\cdot)$ is compact and therefore $S_{2}(t)$ is continuous in the uniform operator topology for $t>0$. Then, $\phi(\tau)$ is also continuous in the uniform operator topology on $(0, a]$. Thus $\| S_{2}\left(t_{2}-t_{1}+\tau\right)-$ $S_{2}(\tau) \|_{\mathscr{L}(\mathbb{X})} \rightarrow 0$ as $t_{2}-t_{1} \rightarrow 0$. Meanwhile, $\phi(\tau)$ is bounded on $[0, a]$. Hence, using Lebesgue dominated convergence theorem we deduce that $\lim _{t_{2}-t_{1} \rightarrow 0} I_{2}=\lim _{t_{2}-t_{1} \rightarrow 0} I_{4}=0$.

Moreover, from (23) we have

$$
\begin{aligned}
& I_{3} \leq M_{1} M_{2}\left\|v_{0}\right\|\left|t_{2}-t_{1}\right|, \\
& I_{5} \leq M_{1} M_{2} a\|\mu\|_{L^{\infty}\left(J, \mathbb{R}^{+}\right)}\left|t_{2}-t_{1}\right| .
\end{aligned}
$$

Hence, $\lim _{t_{2}-t_{1} \rightarrow 0} I_{3}=\lim _{t_{2}-t_{1} \rightarrow 0} I_{5}=0$.

In short, we have show, that $Q\left(B_{r}\right)$ is relatively compact for $t \in J,\left\{Q u: u \in B_{r}\right\}$ is a family of equicontinuous functions. It follows from Ascoli-Arzela's theorem that $Q$ is compact. By Schauder fixed point theorem $Q$ has a fixed point $u \in B_{r}$, which obviously is a mild solution to (1).

\section{An Example}

In order to illustrate our main results, we consider the following initial-boundary value problem, which is a model for elastic system with structural damping

$$
\begin{gathered}
\frac{\partial^{2} u(x, t)}{\partial t^{2}}-\rho \frac{\partial^{3} u(x, t)}{\partial x^{2} \partial t}+\frac{\partial^{4} u(x, t)}{\partial x^{4}} \\
=f(x, t, u(x, t)), \quad(x, t) \in[0,1] \times[0, a], \\
u(0, t)=u(1, t)=0, \quad t \in[0, a], \\
u(x, 0)=\varphi(x), \quad \frac{\partial}{\partial t} u(x, 0)=\psi(x), \quad x \in[0,1],
\end{gathered}
$$

where $a>0, \rho \geq 2$ are all constants, $f:[0,1] \times[0, a] \times \mathbb{R} \rightarrow \mathbb{R}$ is continuous.

Let $\mathbb{X}=L^{p}([0,1], \mathbb{R})(1<p<+\infty)$, we define the linear operator $\mathscr{A}$ in $\mathbb{X}$ by

$$
\mathscr{A} u=-\frac{\partial^{2} u}{\partial x^{2}}, \quad u \in D(\mathscr{A})=W^{2, p}(0,1) \cap W_{0}^{1, p}(0,1) .
$$

It is well known from [13] that $-\mathscr{A}$ is the infinitesimal generator of a $C_{0}$-semigroup $T(t)(t \geq 0)$ on $\mathbb{X}$.

Let $u(t)=u(\cdot, t), f(t, u(t))=f(\cdot, t, u(\cdot, t))$, then the initial-boundary value problem (42) can be reformulated as the following abstract second order evolution equation initial value problem in $\mathbb{X}$ :

$$
\begin{gathered}
\ddot{u}(t)+\rho \mathscr{A} \dot{u}(t)+\mathscr{A}^{2} u(t)=f(t, u(t)), \quad 0<t<a, \\
u(0)=\varphi, \quad \dot{u}(0)=\psi .
\end{gathered}
$$

In order to solve the initial-boundary value problem (42), we also need the following assumptions:

(b1) $\varphi \in W^{2, p}(0,1) \cap W_{0}^{1, p}(0,1), \psi \in L^{p}([0,1], \mathbb{R})$.

(b2) The partial derivative $f_{u}^{\prime}(x, t, u)$ is continuous.

Theorem 6. If the assumptions (b1) and (b2) are satisfied, then for any $\rho \geq 2$, the initial-boundary value problem (42) has a unique mild solution $u \in C\left([0, a], L^{p}([0,1], \mathbb{R})\right)$.

Proof. From the assumptions (b1) and (b2), it is easily seen that the conditions in Theorem 4 are satisfied. Hence, by Theorem 4 , for any $\rho \geq 2$, the problem (44) has a unique mild solution $u \in C([0, a], \mathbb{X})$, which means $u$ is a mild solution for initial-boundary value problem (42).

\section{Acknowledgments}

The authors are grateful to the anonymous referee for his/her valuable comments and suggestions, which improve the presentation of the original paper. Research was supported by NNSFs of China (11261053, 11061031).

\section{References}

[1] G. Chen and D. L. Russell, "A mathematical model for linear elastic systems with structural damping," Quarterly of Applied Mathematics, vol. 39, no. 4, pp. 433-454, 1982.

[2] F. L. Huang, "On the holomorphic property of the semigroup associated with linear elastic systems with structural damping," Acta Mathematica Scientia, vol. 5, no. 3, pp. 271-277, 1985.

[3] F. Huang, "A problem for linear elastic systems with structural damping," Acta Math-Ematica Scientia, vol. 6, no. 1, pp. 101-107, 1986 (Chinese).

[4] S. Chen and R. Triggiani, "Proof of extensions of two conjectures on structural damping for elastic systems: the systems: the case $1 / 2 \leq \alpha \leq 1$," Pacific Journal of Mathematics, vol. 136, no. 1, pp. $15-55,1989$.

[5] S. Chen and R. Triggiani, "Gevrey class semigroups arising from elastic systems with gentle dissipation: the case $0<\alpha<1 / 2$," Proceedings of the American Mathmematical Society, vol. 110, no. 2, pp. 401-415, 1990. 
[6] F. L. Huang, "On the mathematical model for linear elastic systems with analytic damping," SIAM Journal on Control and Optimization, vol. 26, no. 3, pp. 714-724, 1988.

[7] K. Liu and Z. Liu, "Analyticity and differentiability of semigroups associated with elastic systems with damping and gyroscopic forces," Journal of Differential Equations, vol. 141, no. 2, pp. 340-355, 1997.

[8] F. L. Huang and K. S. Liu, "Holomorphic property and exponential stability of the semigroup associated with linear elastic systems with damping," Annals of Differential Equations, vol. 4, no. 4, pp. 411-424, 1988.

[9] F. L. Huang, Y. Z. Huang, and F. M. Guo, "Holomorphic and differentiable properties of the $C_{0}$-semigroup associated with the Euler-Bernoulli beam equations with structural damping," Science in China A, vol. 35, no. 5, pp. 547-560, 1992.

[10] F. L. Huang, K. S. Liu, and G. Chen, "Differentiability of the semigroup associated with a structural damping model," in Proceedings of the 28th IEEE Conference on Decision and Control (IEEE-CDC 1989), pp. 2034-2038, Tampa, Fla, USA, 1989.

[11] K.-J. Engel and R. Nagel, One-Parameter Semigroups for Linear Evolution Equations, Springer, New York, NY, USA, 2000.

[12] A. Pazy, Semigroups of Linear Operators and Applications to Partial Differential Equations, Springer, Berlin, Germany, 1983.

[13] D. Henry, Geometric Theory of Semilinear Parabolic Equations, Springer, New York, NY, USA, 1981. 


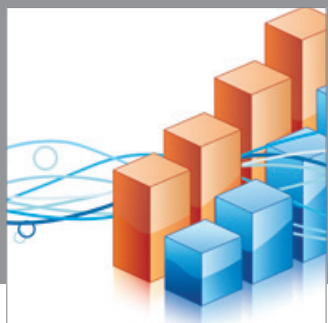

Advances in

Operations Research

mansans

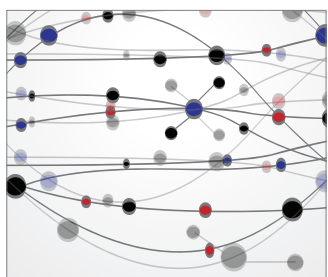

The Scientific World Journal
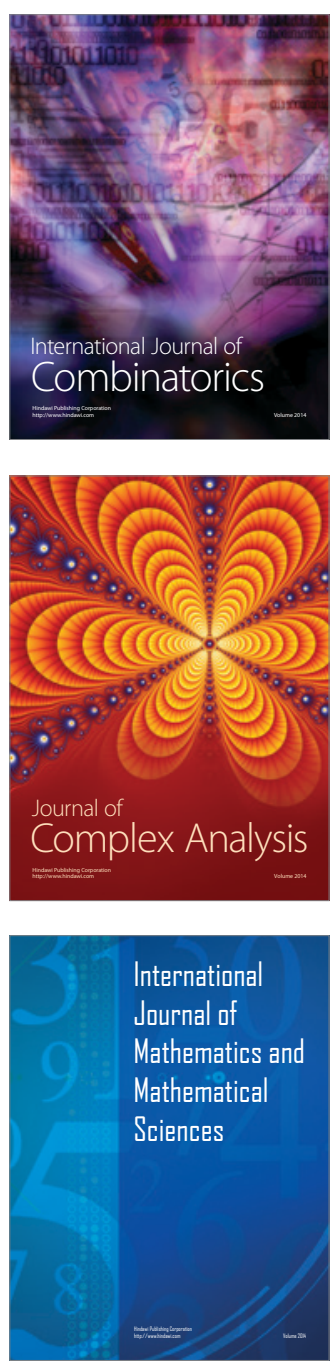
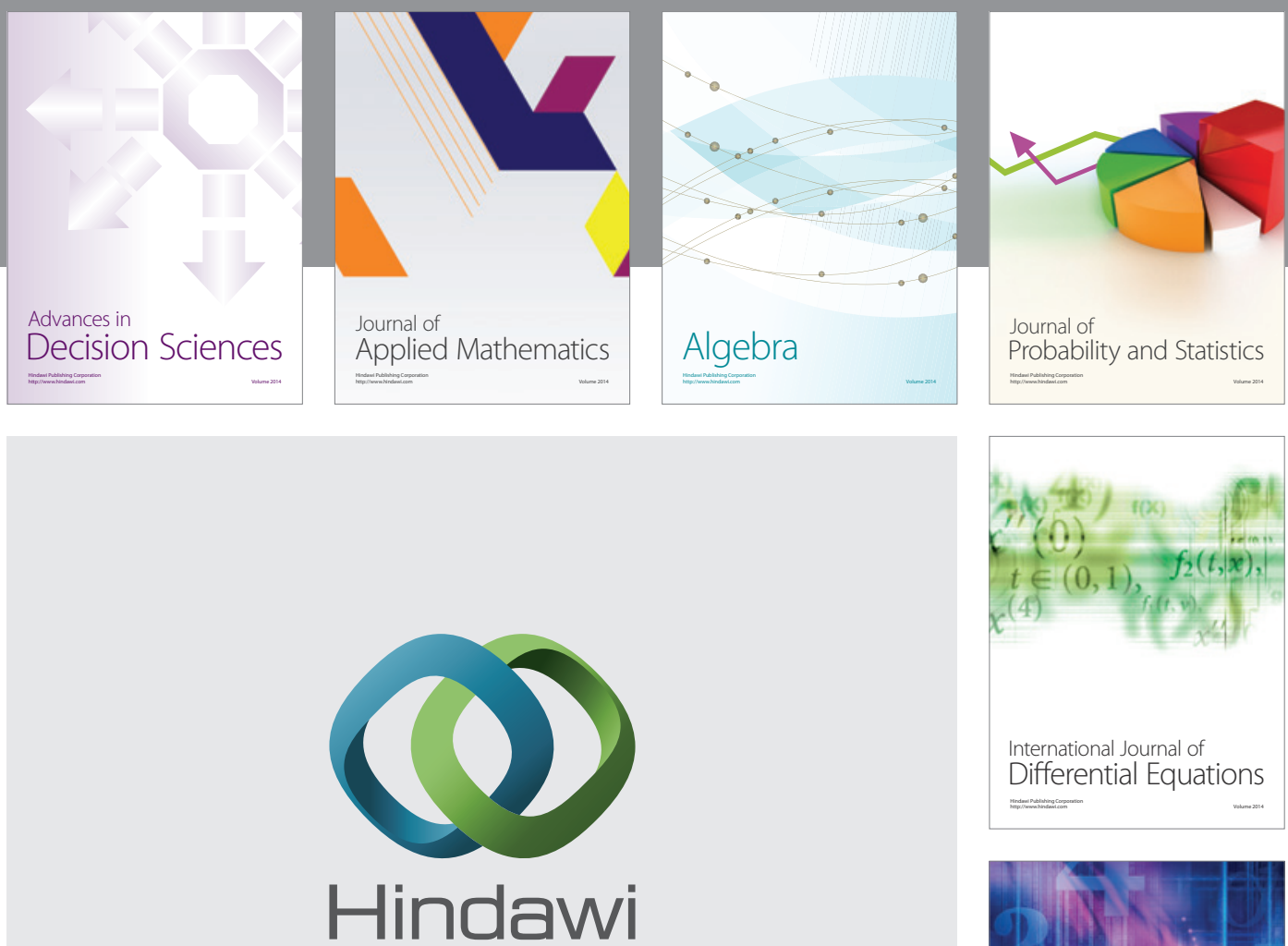

Submit your manuscripts at http://www.hindawi.com
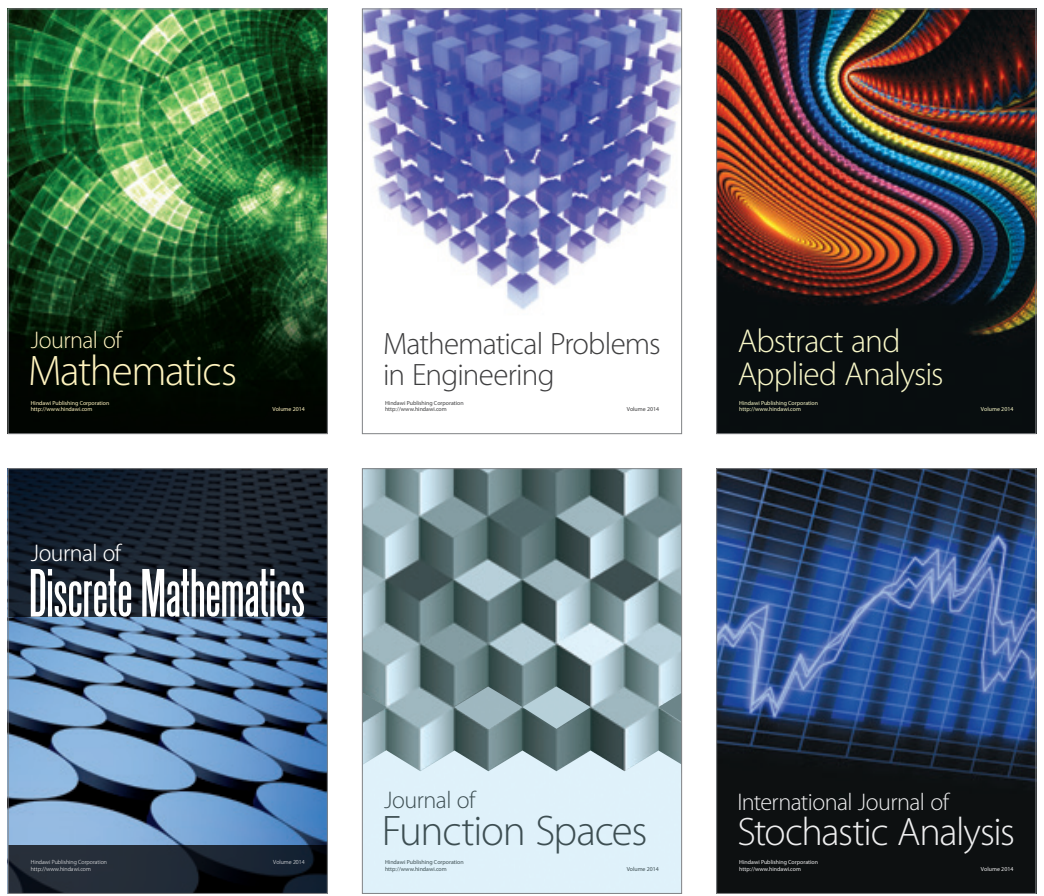

Journal of

Function Spaces

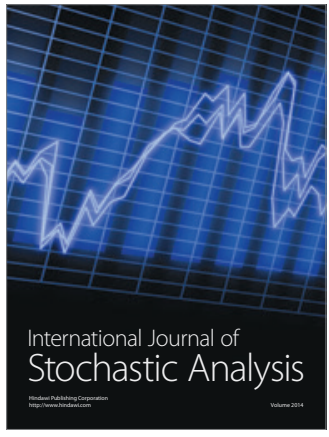

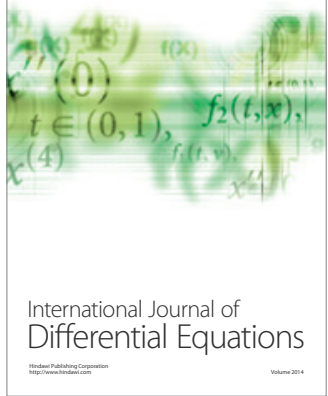
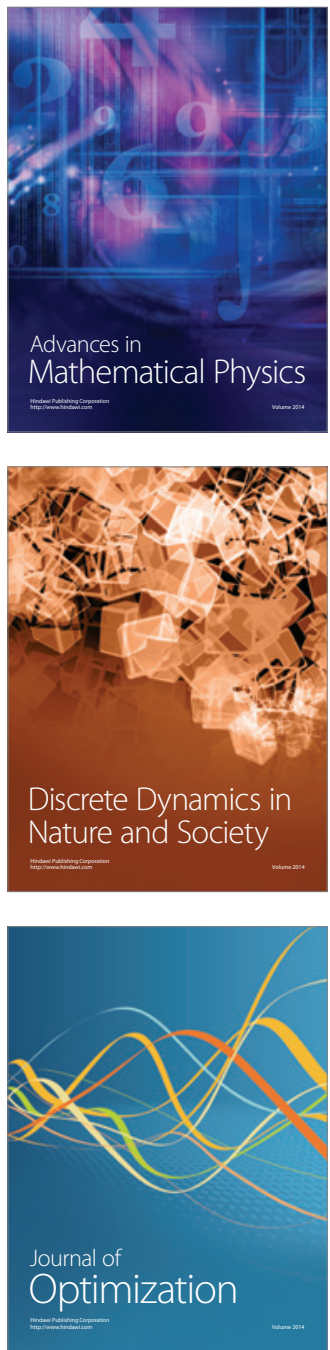